\title{
Hochempfindliche Stahlmembran-Drucksensoren mit lasergerechtem Messbrückenlayout
}

\author{
Günter Schultes, Dirk Göttel, Mario Cerino, \\ guenter.schultes@htw-saarland.de \\ ZeMA gGmbH, Zentrum für Mechatronik und Automatisierungstechnik, \\ 66121 Saarbrücken, Deutschland
}

\begin{abstract}
Stahlmembran-Drucksensoren zur Messung hydraulischer Drücke haben eine beachtliche technologische Reife erreicht und werden bereits in sehr hohen Stückzahlen für unterschiedliche Industriezweige produziert. Dennoch bieten die Sensorelemente ein hohes Optimierungspotential. Diese Arbeit zeigt Innovationsschritte für die sensorisch aktive Dünnschicht, deren Strukturierung und Kontaktierung auf. Eine hochempfindliche Sensorschicht liefert ein Vielfaches des üblichen Signals. Die Strukturierung der Dünnschicht erfolgt mit Hilfe eines Ultrakurzzeitlasers, der einige wenige Trenngräben direkt in die Schicht schneidet. Ein topologisch neuartiges Brückendesign gestattet es, die Vorteile der Laserstrukturierung auszuschöpfen. Da die Sensorschicht direkt durch Draht-Ultraschall Bonden kontaktierbar ist, werden Kontaktschichten und sämtliche photolithographische Prozessschritte überflüssig. Durch den Verzicht auf Ätzmedien können Defekte durch Migrationsprozesse ausgeschlossen werden. Es entsteht ein optimiertes Sensorelement mit höheren Spezifikationen und prozesstechnischen Vereinfachungen, die sich zur Kostenreduktion nutzen lassen.
\end{abstract}

Key words: hochempfindliche Drucksensoren, DMS, Laserstrukturierung, Dünnschichtschaltung

\section{Einleitung}

Drucksensoren aus Edelstahlmesselementen werden vielmillionenfach in der Industrie produziert. Typische Anwendungen finden sich in Steuerungsanlagen von Einspritz- und Bremssystemen, in Getrieben sowie in Hydraulik- und Chemieanlagen. Bedingt durch den immer weiter zunehmenden Bedarf an Steuerungs- und Automatisierungskomponenten steigen die jährlich produzierten Stückzahlen kontinuierlich an. Durch die Massenfertigung werden günstige Stückpreise erzielt, was wiederum die Möglichkeit des Einsatzes in neue Bereiche eröffnet.

Die Grundanordnung eines StahlmembranDrucksensors ist in Fig. 1 wiedergegeben. Der zu messende hydraulische oder pneumatische Druck wirkt auf eine Membran, die eine Isolierschicht und eine Messanordnung aus dünnen Sensorfunktionsschichten trägt. Eine Finite Elemente Simulation (FEM) nach Fig. 2 zeigt die mechanischen Spannungen der topfförmigen Geometrie des Sensors, wenn dieser mit Druck beaufschlagt wird. Die stärksten Spannungen entstehen am Übergang von der Bohrung zur Membran und im Zentrum. An diesen Stellen ergeben sich als erstes plastische Verformungen, die bei einem gezielten Überlastungsprozess, einer sogenannte Autofrettage, hervorgerufen werden, um den Sensor zu stabi-

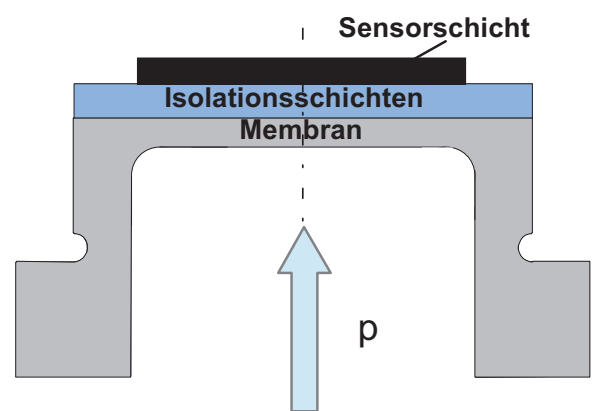

Fig. 1 Schema eines Stahlmembran-Drucksensors Der Sensorkörper aus Edelstahl schließt mit einer Membran ab auf der die Dünnschichten appliziert sind.

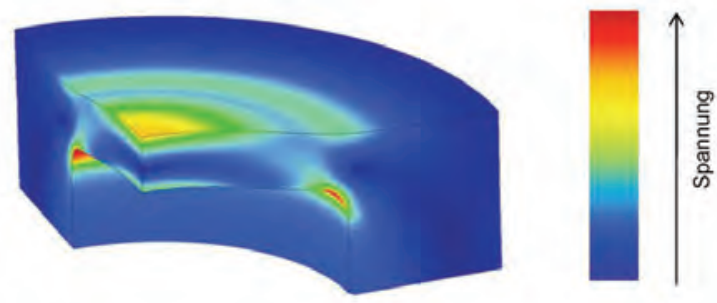

Fig. 2 Ergebnis einer FEM-Simulation eines Drucksensors. Dargestellt ist die mechanische Spannung unter Druck. In der Membranmitte stellt sich an der Oberseite eine Dehnung ein, während die Stauchungszone weiter außen an der Bohrungskante liegt. Quelle: www.drucksensor-knowhow, Fa. Wika. 
lisieren. An der Membranoberseite entsteht bei Druckbelastung eine reversible Deformation sowohl in tangentialer als auch in radialer Richtung. Im Folgenden wird nur auf die radiale Deformation eingegangen. Um das Membranzentrum herum liegt eine radial ausgerichtete Dehnungszone (Zug) vor, weiter außen an der Membrankante befindet sich eine radiale Stauchungszone (Druck).
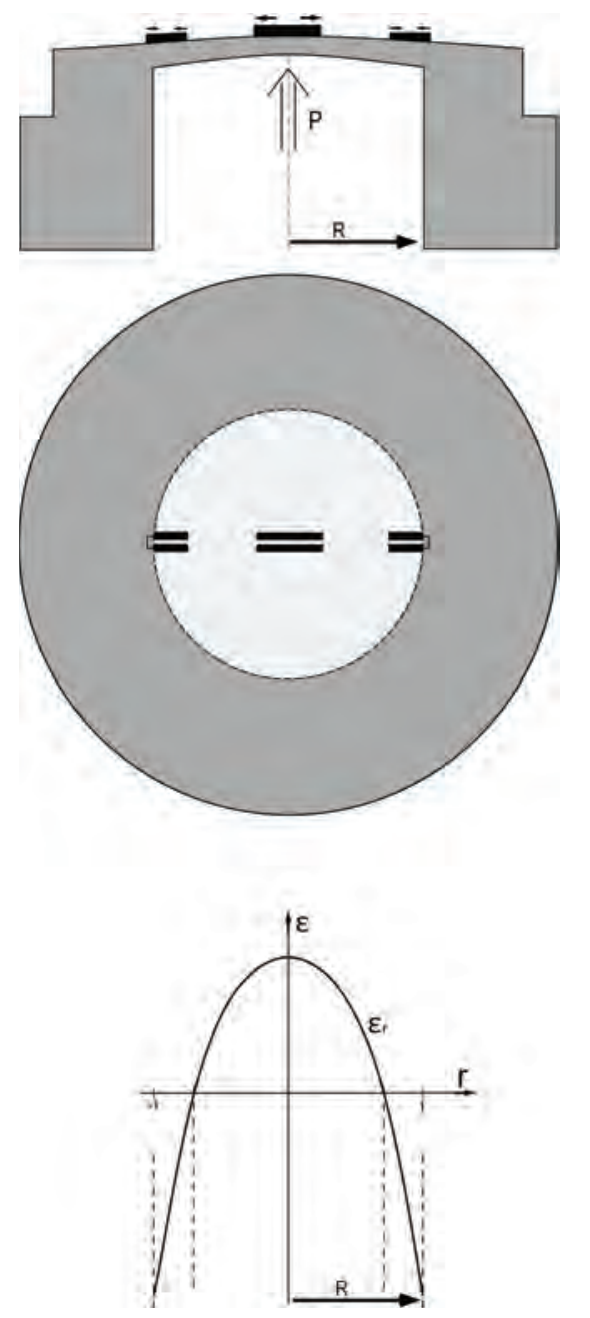

Fig. 3 Überhöht eingezeichnete Auswölbung der Membran. Die Zone um das Zentrum ist in radialer Richtung gedehnt, am Rand ist sie gestaucht. DMS sind entsprechend platziert. Das Diagramm gibt lediglich die radiale Deformation an.

Fig. 3 zeigt die geometrisch überzeichnete Auswölbung der Membran, auf der bereits Dehnungsmesswiderstände (DMS) für die Messung der radialen Dehnung im Zentrum und solche zur Messung der Stauchung an der Membrankante eingezeichnet sind. Der parabelförmige Verlauf der radialen Deformation ist ebenfalls angegeben [siehe z.B. 1]. Zwischen der Dehnung im Zentrum und dem Stauchungsring verläuft eine neutrale Zone.
Mechanisch wird die Sensormembran zumeist auf eine Beanspruchung von etwa ein Promille Dehnung ausgelegt, welche beim vorgesehenen Nenndruck erreicht wird. Dies geschieht im wesentlichen durch die Anpassung der Membrandicke an die jeweiligen Nenndrücke von etwa 25 bis 2000 bar. Übliche Abmaße liegen bei Außendurchmessern von $8 \mathrm{~mm}$ und Membrandurchmessern von etwa $4 \mathrm{~mm}$.

Die Produktion der Sensorelemente erfordert zunächst eine Politur der Membran und die Abscheidung einer elektrischen Isolierschicht von bis zu $8 \mu \mathrm{m}$ Dicke, die für Durchschlagsspannungen bis zu 1000 VDC ausgelegt ist. Dann wird eine dehnungsempfindliche Funktionsschicht, üblicherweise eine CrNi-Legierung, aufgesputtert, aus der photolithographisch eine Brückenschaltung mit mäanderförmigen DMS strukturiert wird. Weiterhin bringt man meist drei verschiedene Kontaktschichten auf, die ebenfalls photolithographische Strukturierungen erfahren. Insgesamt ist eine Vielzahl von Prozessschritten notwendig, bis eine Sensorzelle fertig gestellt ist.

\section{Konzeption, Versuche und Ergebnisse}

Unsere Konzeption übersteigt den beschriebenen Stand der Technik in verschiedenen Punkten.

- Eine nickelhaltige Kohlenstoff Funktionsschicht (Ni:a-C:H oder nanoNi@C) liefert eine hohe Empfindlichkeit von fast $30 \mathrm{mV} / \mathrm{V}$ gegenüber $2 \mathrm{mV} / \mathrm{V}$ von $\mathrm{CrNi}$-Schichten.

- Ein neuartiges Messbrückenlayout wird per UV-Ultrakurzzeitlaser in die Funktionsschicht geschrieben. Dadurch werden alle photolithographischen und chemischen Ätzprozesse obsolet.

- Die elektrische Kontaktierung erfolgt durch Bonden direkt auf der Funktionsschicht; spezielle Kontaktschichten sind nicht mehr nötig.

- Die Anzahl der Fertigungsschritte wird deutlich reduziert, sodass sich Kostenvorteile ergeben.

Die Eigenschaften von hochempfindlichen Dünnschichten aus Nickel und Kohlenstoff sind bereits in mehreren Aufsätzen [2, 3] publiziert. Mit Hilfe der Lift-Off Technik lässt sich die neue Funktionsschicht auf Drucksensoren ebenfalls photolithographisch zu einer Brückenschaltung strukturieren [3, 4]. Man erhält auf diese Weise eine um den Faktor 15 gesteigerte Empfindlichkeit. Die Herstellung ist ansonsten analog zum oben beschriebenen Stand der Technik. 
Im vorliegenden Aufsatz wird stattdessen eine direktschreibende Strukturierung der Sensorschicht beschrieben, die komplett ohne photolithographische Prozesse auskommt. Die Laserstrukturierung findet nach der vollflächigen Beschichtung der Messzellenmembran mit der Funktionsschicht Ni:a-C:H von etwa 140 bis $250 \mathrm{~nm}$ Schichtdicke statt. Ein Lasersystem der Firma 3D-Micromac (microSTRUCT-PV) mit einer Strahlquelle der Firma Lumera kommt zum Einsatz. Diese Laserquelle emittiert ultrakurze Pulse von weniger als 15 ps Dauer bei der UV-Wellenlänge von $355 \mathrm{~nm}$. In Versuchsreihen konnten Prozessparameter identifiziert werden, die es gestatten, lediglich die Funktionsschicht abzulösen ohne Beschädigungen der darunter befindlichen Isolierschicht hervorzurufen. Auf diese Weise können Trennschnitte in die Funktionsschicht geschrieben werden, so dass voneinander elektrisch isolierte Zonen entstehen.

Fig. 4 zeigt schematisch den Vorgang des Laserschneidens. Die Schnittbreite des auf die Schichtebene fokussierten Strahls beträgt etwa 5 bis $10 \mu \mathrm{m}$. Der Schnittgraben lässt sich durch eine spiralartige Bewegung des Strahls oder durch überlappende Kreise verbreitern. Der Laser wird mit Hilfe eines Ablenkspiegelsystems so über die Oberfläche der Sensorschicht geführt, dass der Laserstrahl die Bereiche überstreicht, in denen die Sensorschicht unterbrochen werden soll. Die Schicht absorbiert die Energie des Lasers und wird dabei abgetragen. Die gewünschte Struktur lässt sich somit direkt in die Sensorschicht schreiben.

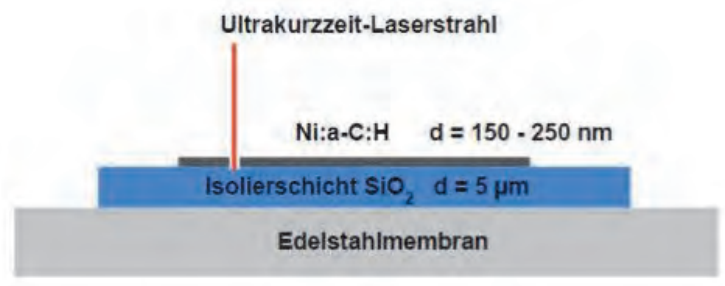

Fig. 4 Die Ultrakurzzeitlaserstrahlung mit $355 \mathrm{~nm}$ und $<15$ ps Pulsdauer trennt lediglich die dünne Ni:a-C:H-Schicht. Die Isolierschicht aus $\mathrm{SiO}_{2}$ bleibt unverletzt. Auf diese Weise lässt sich eine Brückenschaltung direkt in die Funktionsschicht schreiben.

In Fig. 5 sind exemplarisch Ergebnisse der Laserschnittversuche gezeigt. Misst man mit einem taktilen Oberflächenprofilometer quer zum Schnitt (Fig. 6), kann verifiziert werden, dass bei korrekt eingestellten Laserparametern lediglich die Funktionsschicht abgetragen wird, während die darunter befindliche Isolierschicht unbeschädigt bleibt.

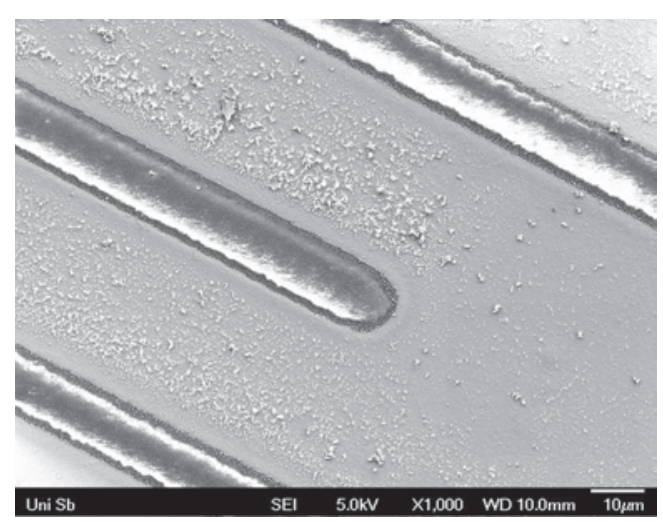

Fig. 5 Elektronenmikroskopische Aufnahme von Laserschnitten in die Sensorfunktionsschicht. Die Ablationsreste müssen durch einen Reinigungsschritt entfernt werden.

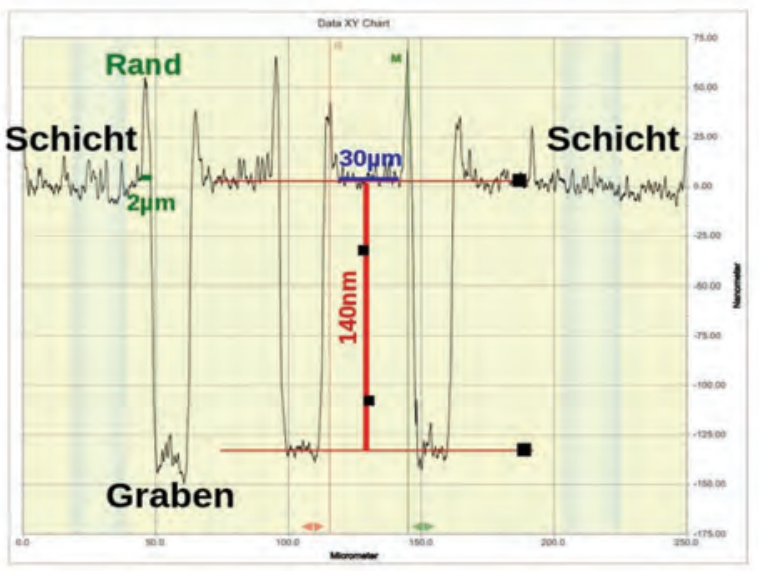

Fig. 6 Profil der Schnitte. Nur die Funktionsschicht $(140 \mathrm{~nm})$ ist entfernt, der Schnitt taucht nicht in die Isolierschicht ein.

Die direkte Ablation der Funktionsschicht durch Ultrakurzzeitlaser eröffnet die Möglichkeit einer direkt schreibenden Strukturierung [6]. Man könnte übliche Brückenlayouts ohne photolithographische Umwege in die Schicht schneiden, in dem der Laserstrahl einer Schraffur entsprechend über die abzutragenden Bereiche geführt wird.

Eine intelligentere Vorgehensweise kommt hingegen mit einem Minimum an Schnitten aus, um ein Brückenlayout zu kreieren. Dazu wird das einfache Schnittmuster der Fig. 7 auf eine Membran geschrieben. 


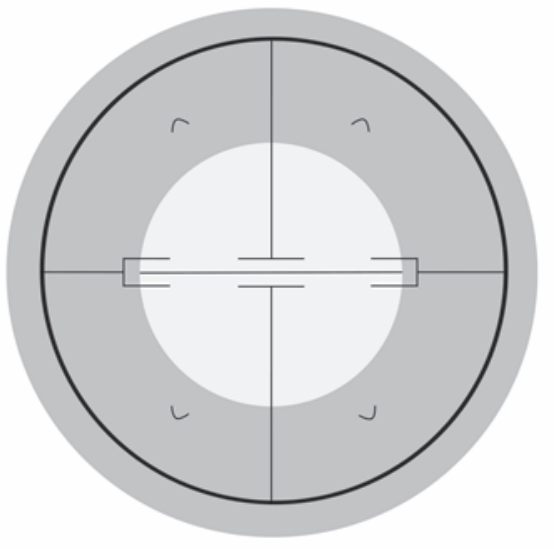

Fig. 7 Neuartiges Schnittmuster zum Erzeugen einer Wheatstone'schen Messbrücke. Die eigentliche Membran ist wie in Fig. 3 hell gehalten. An den vier Positionsmarken erfolgt die Spannungsversorgung und der Signalabgriff, jeweils diagonal gegenüber. bunden sind. Die Widerstände sind durch enge Pfade definiert, je ein enger Pfad verbindet die benachbarten Segmente der Brücke. Die beiden U-förmigen Widerstände am Rand der Membran bilden die Widerstände R1 und R2, während die geraden Pfade in der Mitte die Widerstände R3 und R4 bilden. Durch das Längen- zu Breitenverhältnis der Pfade wird die Anzahl der Quadratwiderstände (Rsq) eingestellt. Im Beispiel gehen wir von einem Flächenwiderstand von $150 \Omega / s q$ aus. Die Widerstände R1 bis R4 weisen eine Länge von $1 \mathrm{~mm}$ und eine Breite von etwa $30 \mu \mathrm{m}$ auf. Somit werden etwa 33 Rsq realisiert, die mit dem angegebenen Flächenwiderstand etwa $5 \mathrm{k} \Omega$ für jeden Widerstand ergeben. Mit den segmentgroßen Zuleitungen ergeben sich Brückenwiderstände von etwa $5,3 \mathrm{k} \Omega$. Die Zuleitungen von den Positionsmarken bis zu je zwei Widerständen entsprechen etwa 1 Rsq, d.h. der Zuleitungswiderstand ist so gering wie möglich. Man kommt deshalb ohne weitere Metallisierung der Zuleitungszonen aus.
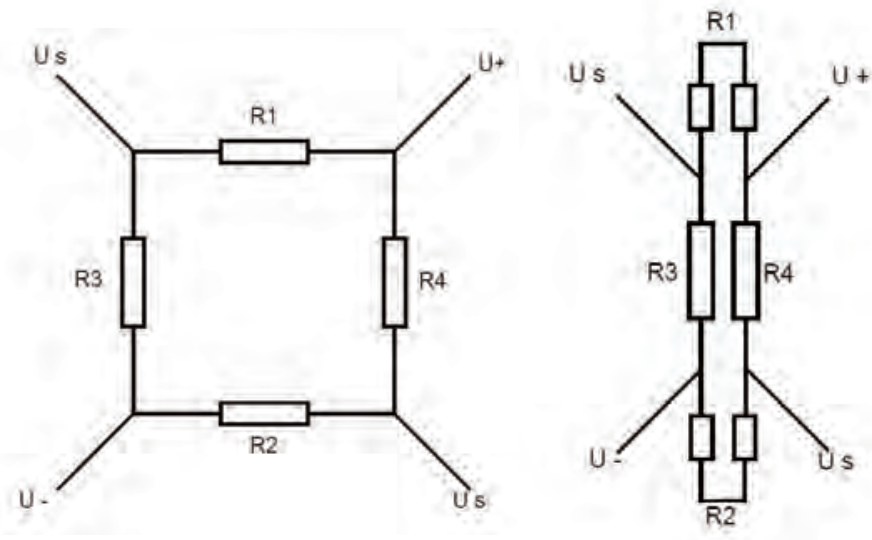

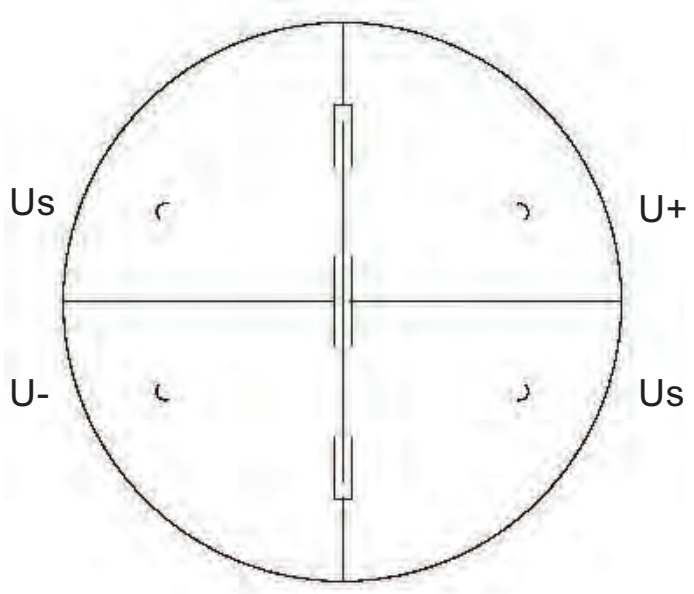

Entwicklung der Schnittanordung aus der Brückenschaltung

Fig. 8 Aus der üblichen Darstellung einer Wheatstone'schen Brückenschaltung wird ein Schnittmuster entwickelt. Der große Kreis im rechten Bild isoliert die elektrische Dünnschichtschaltung von der Umgebung. An den Markierungen U+ und U- schließt man die Versorgungsspannung an, während Kontakte an den Markierungen Us die Brückenspannung, also die vom Druck auf die Membran abhängige Sensorspannung liefert.

In Fig. 8 kann die Entstehung und Wirkungsweise des neuen Layouts nachvollzogen werden. Die Brückenschaltung wird umgezeichnet und als Schnittlinien in eine elektrisch leitende Schicht eingeschrieben. Die Membranfläche sei zunächst vollflächig beschichtet. Als erstes isoliert man durch einen Laserschnitt in Form eines Kreises die Schaltung von der Umgebung. Der innen liegende aktive Bereich der Sensorschicht wird in vier Segmente unterteilt, wobei jeweils zwei benachbarte Segmente über einen Widerstand miteinander elektrisch ver-
Die vorgeschlagene Messbrückenstruktur kommt mit einer im Vergleich zu den bekannten Strukturen geringeren Anzahl an zu schreibenden Linien aus, da weder Zuleitungen, noch mäanderförmige Widerstände ausgebildet werden müssen. Die reduzierte Anzahl an zu schreibenden Linien ermöglicht es, die Struktur in kürzerer Zeit mit dem Laser zu schreiben.

Die elektrische Kontaktierung erfolgt direkt durch Al-Draht Bonden auf die Funktionsschicht aus Ni:a-C:H. Das Aufbringen und Strukturieren 
von zusätzlichen Kontaktschichten ist überflüssig. Unter Verwendung eines Dünndrahtbonders der Firma TPT (Typ HB16) wurden per Wedge-Ultraschall Verfahren $50 \mu \mathrm{m}$ dicke AlSi1\%-Drähte auf die Messzelle gebondet. Zur Beurteilung der Qualität der Bondverbindungen wird die in [5] beschriebene zerstörende Zugprüfung (Pulltest) angewendet. Alle 33 getesteten Verbindungen zeigen Abreißkräfte oberhalb der minimal geforderten Kraft von $11 \mathrm{cN}$.

An dieser Stelle sei auf einen weiteren Vorteil des neuen Brückenlayouts hingewiesen. Wenn zunächst die beiden Bondkontakte für die Spannungsversorgung an den Positionsmarken hergestellt werden und eine Spannung angeschlossen wird, kann durch Messen mittels Nadeln in den Us-Segmenten die richtige Position für eine ausgeglichene Brücke ermittelt werden. Wird der Drahtbond in einem Segment nicht an einer Stelle angeordnet, die von beiden an das Segment grenzenden Widerständen gleich weit entfernt ist, so wird der elektrische Widerstand vom Bondkontakt zu einem der angrenzenden Widerstände größer, als zum anderen Widerstand. Kleine Abweichungen in den Werten des elektrischen Widerstands der an das Segment angrenzenden Widerstände können demnach durch geeignete Wahl der Position des Bondkontakts ausgeglichen werden.

Fig. 9 zeigt eine per Laser strukturierte Messzelle mit den elektrischen Bondkontakten. Zur Zeit ist es uns nicht möglich, die Messzelle auf einen Gewindestutzen zu schweißen und mit hydraulischem Druck zu belasten. Um dennoch die Funktionsweise und die Empfindlichkeit der laserstrukturierten Messzellen bis 6 bar zu bestimmen, werden die strukturierten und elektrisch kontaktierten Messzellen in eine Verschraubung integriert, an $10 \mathrm{~V}$ Versorgungsspannung angeschlossen und das Brückennullsignal mit einem Messgerät (Agilent 34401A) bestimmt. Die angeschlossene Druckluft wird in 0,8 bar Schritten bis auf 6 bar erhöht und das Brückensignal bestimmt. Durch Auftragen des Brückensignals in Abhängigkeit der Drücke, kann die Empfindlichkeit aus der resultierenden Geraden ermittelt werden.

In Fig. 10 ist ein typisches Versuchsergebnis gezeigt. Von der mechanischen Auslegung handelt es sich um eine Messzelle für 100 bar bei Verwendung von CrNi-DMS. Die Membran wurde wie beschrieben mit der hochempfindlichen Ni:a-C-H beschichtet, laserstrukturiert und gebondet. Schon bei 6 bar ist eine Empfindlichkeit von etwa 1,7 mV/V zu verzeichnen, hochgerechnet auf die Nennlast von 100 bar bedeutet dies eine Empfindlichkeit von 30 $\mathrm{mV} / \mathrm{V}$. Zum Vergleich ist der Verlauf des Signals bei Verwendung von standardmäßigen CrNi-DMS angegeben. Die korrekte Arbeits-

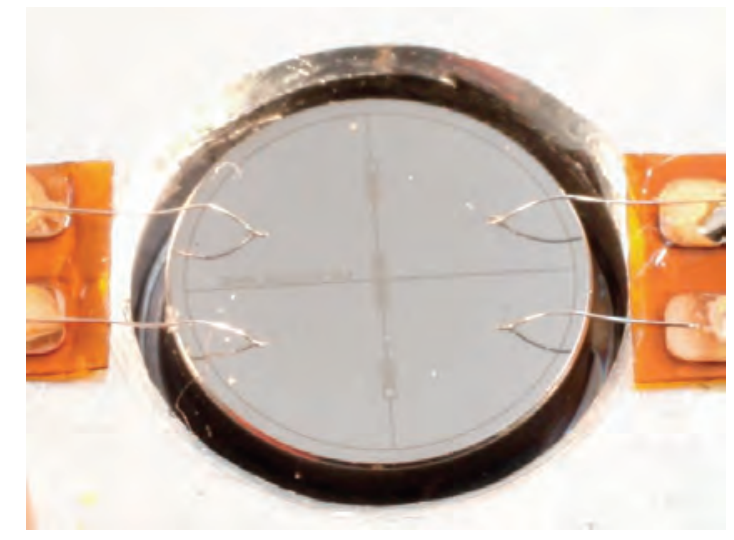

Fig. 9 Die laserstrukturierte Messzelle in einer Halterung. Man erkennt das vorgestellte Brückenlayout und die durch Wedge-Bonden hergestellten Al-Bondverbindungen. Weiter außen sind die Potentiale durch angelötete Litzen weitergeführt.

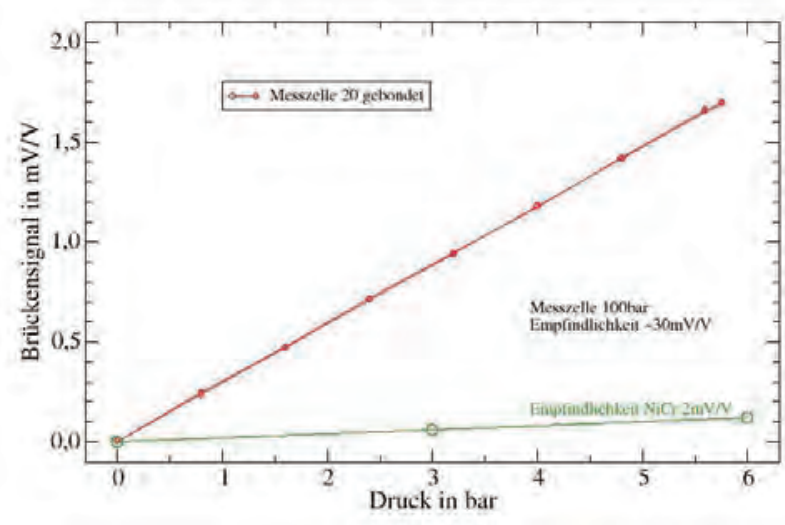

Fig. 10 Signalverlauf an einer Messzelle bis zu einem pneumatischen Druck von 6 bar. Hochgerechnet auf den Nenndruck von 100 bar beträgt die Empfindlichkeit etwa $30 \mathrm{mV} / \mathrm{V}$.

weise der neuartigen Drucksensorzellen ist somit gezeigt.

Durch den Wegfall sämtlicher Lithographie- und Ätzprozesse und durch das einfache und sichere Bonden auf der hochempfindlichen Funktionsschicht werden bedeutende prozesstechnische Vereinfachungen mit entsprechenden Kostenvorteilen ermöglicht. Zusätzlich ist eine Reduktion von Feldausfällen zu erwarten, da während der Herstellung keine Ätzmedien erforderlich sind und folglich keine lonen verschleppt werden, die durch Migration Ausfälle verursachen können.

Verglichen mit dem derzeitigen Stand der Technik fallen bei unserer Vorgehensweise viele Schritte weg. Dies sind konkret die Beschichtungsprozesse mit meist drei verschiedenen Schichten zum Aufbau der Kontaktgebung und deren komplette photolithographische Strukturierung. Außerdem fällt die 
Lithographie der Funktionsschicht weg. Es gibt also weniger Beschichtungsprozesse und keinerlei Photolithographie bestehend aus der meist zweifach wiederholten Belackung mit Photoresist, dem Belichten, Entwickeln, Ätzen und Reinigen. Entsprechende Kostenvorteile dürften auf der Hand liegen.

\section{Zusammenfassung und Ausblick}

In dieser Arbeit wird ein topologisch neuartiges Layout für eine vollständige Brückenschaltung vorgestellt. Diese Dünnschichtschaltung erfordert nur ein Minimum an Laserschnitten und verzichtet ganz auf das flächige Abtragen von vorher aufgebrachten Schichtpartien. Somit ist die vorgestellte Struktur laserfreundlich und die entsprechende Fertigung ist lasergerecht. Da die Struktur zudem leicht durch direkte Trennschnitte mit einem Ultrakurzzeitlaser realisiert wird, ergeben sich erhebliche Vorteile für die gesamte Herstellungskette. An vorbestimmten Stellen erfolgt eine elektrische Kontaktierung durch direktes Al-Draht Bonden auf der Funktionsschicht. Diese neuartige Vorgehensweise ist prinzipiell nicht an die Verwendung von hochempfindlichen Funktionsschichten gebunden, sondern auch mit den heute üblichen $\mathrm{CrNi}$ Schichten möglich. Eine um den Faktor von etwa 15 gesteigerte Empfindlichkeit ergibt sich natürlich nur bei Verwendung der hochempfindlichen Ni:a-C:H Schicht.

Als nächstes gilt es komplette Sensoren, bestehend aus einer Messzelle und einem Gewindekörper aufzubauen und zu testen. Dazu muss der Schweißprozess der beiden Körper realisiert werden, sodass über den gesamten Druckbereich gemessen werden kann. Auch wird eine dünne Passivierungsschicht notwendig sein, wobei zu ergründen ist, ob der Lasertrenn- und Trimmprozess durch eine solche Schicht erfolgen kann. Alsdann werden die Sensoren durch Langzeittests unter Temperatur und Druck zu testen sein.

Insgesamt ergeben sich durch die Verwendung einer hochempfindlichen Sensorschicht, durch die beschriebene topologisch neue Messbrücke und durch den Verzicht auf sämtliche Lithographie und Ätzprozesse erhebliche Vorteile auf dem Gebiet der Stahlmembran-Drucksensoren.

\section{Literatur}

[1] Hütte, Das Ingenieurwesen, Springer Verlag

[2] R. Koppert, S. Uhlig, H. Schmid-Engel, D. Göttel, A.-C. Probst, G. Schultes, U. Werner; Structural and physical properties of highly piezoresistive nickel containing hydrogenated carbon thin films; Diamond and Related Materials;vol. 25; pp. 5058, 2012

[3] R. Koppert, D. Göttel, G. Schultes, U. Werner; NanoNi@C: Hochempfindliche Funktionsschicht für Druck- und Kraftsensoren; tm - Technisches Messen: Vol. 77, 2010, No. 12, 631-637, doi: 10.1524/teme.2010.0111

[4] Ralf Koppert, Dissertation, Nickelhaltige Kohlenwasserstofffunktionsschichten (Ni:a-C:H) für Drucksensoren, ISBN 978-3-8322-9708-4

[5] Deutscher Verband für Schweißtechnik e. V.; Prüfverfahren für Drahtbondverbindungen, Merkblatt DVS 2811, 1996

[6] J.F. Düsing, O. Suttmann, R. Bandorf, H. Gerdes; Oberflächen-Dünnschichtsensorik erschließt neue Anwendungen; Laser Magazin 3/2011. 